\title{
Aseptic Cavernosal Abscess: An Unrecognized Feature of Neutrophilic Dermatosis
}

\author{
Yu Akagi ${ }^{1}$, Yuri Yamagiwa ${ }^{2}$, Harumi Shirai ${ }^{2}$, Takeshi Suzuki ${ }^{2}$, Ibuki Tsuru ${ }^{3}$, Akira Ishikawa ${ }^{3}$, \\ Naoki Akiyama ${ }^{4}$, Mizuki Ogura ${ }^{5}$, Kanae Kobayashi ${ }^{6}$, Yuan Bae ${ }^{7}$, Yamato Suemitsu ${ }^{7}$, \\ Sumihisa Imakado ${ }^{4}$, Momoko Mawatari ${ }^{1}$ and Akihiro Ueda ${ }^{1}$
}

\begin{abstract}
:
A 50-year-old man developed a sterile cavernosal abscess followed by prominent features of necrotizing neutrophilic dermatosis. We conducted a literature review, which revealed that aseptic abscesses in the corpus cavernosum occur in association with neutrophilic dermatosis. Patients with this condition frequently receive unnecessary antibiotic treatment and surgical interventions. Although this condition responds to systemic corticosteroids, the functional prognosis of the penis is poor. Abscess formation may be the initial presentation of neutrophilic dermatoses, and underlying conditions may even be absent. Clinicians need to be aware of this condition to distinguish it from bacterial infection and initiate early disease-specific treatments.
\end{abstract}

Key words: aseptic abscess, corpus cavernosum, neutrophilic dermatosis, pyoderma gangrenosum, corticosteroids

(Intern Med Advance Publication)

(DOI: 10.2169/internalmedicine.7994-21)

\section{Introduction}

An abscess in the corpus cavernosum is a rare clinical entity. It has been reported in the context of infectious diseases and is sometimes preceded by penile trauma (1-3). However, it has rarely been described in the context of non-infectious diseases, and whether or not a cavernosal abscess can be non-infectious remains unclear.

Non-infectious abscesses, also referred to as aseptic abscesses, are typically seen in other organs, usually the spleen and liver, and are associated with various inflammatory conditions. The most frequent underlying condition is inflammatory bowel disease (4). Neutrophilic dermatoses, which are also associated with aseptic abscesses, manifest as a wide range of diseases. Among these, necrotizing neutrophilic dermatosis (NND) shows prominent necrotic cutaneous involvement that is clinically indistinguishable from a necrotizing soft tissue infection. This condition responds dramatically to glucocorticoids, similar to other neutrophilic dermatoses (5). However, cavernosal abscesses have not yet been established as complications of these inflammatory conditions.

We herein report a case of a cavernosal abscess preceding a diagnosis of NND along with a literature review to validate the association between cavernosal abscesses and neutrophilic dermatoses.

\section{Case Report}

The patient was a previously healthy 50 -year-old man. He presented to our hospital with a fever. At presentation, he was not in any acute distress, but his body temperature was $39.1^{\circ} \mathrm{C}$. A complete physical examination was unremarkable except for vague perineal pain. A peripheral blood analysis showed a white blood cell count of $54,110 / \mu \mathrm{L}$ with $89 \%$

\footnotetext{
${ }^{1}$ Department of Infectious Diseases, Japanese Red Cross Medical Center, Japan, ${ }^{2}$ Department of Allergology and Rheumatology, Japanese Red Cross Medical Center, Japan, ${ }^{3}$ Department of Urology, Japanese Red Cross Medical Center, Japan, ${ }^{4}$ Department of Dermatology, Japanese Red Cross Medical Center, Japan, ${ }^{5}$ Department of Hematology, Japanese Red Cross Medical Center, Japan, ${ }^{6}$ Department of Ophthalmology, Japanese Red Cross Medical Center, Japan and ${ }^{7}$ Department of Pathology, Japanese Red Cross Medical Center, Japan Received: May 18, 2021; Accepted: July 8, 2021; Advance Publication by J-STAGE: September 4, 2021 Correspondence to Dr. Yu Akagi, yu.akagi1115@gmail.com
} 

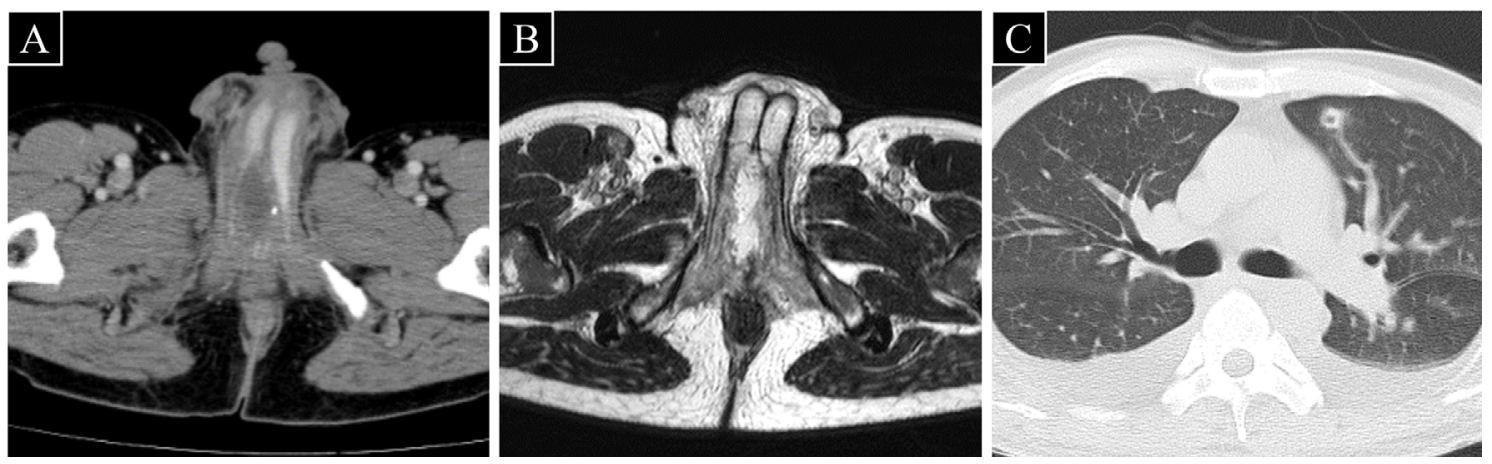

Figure 1. Computed tomography (CT) and magnetic resonance imaging (MRI) findinsg of the patient. Vaguely demarcated low-density area in the right corpus cavernosum visible on contrast-enhanced CT at admission (Panel A). T2-weighted MRI performed on day 2 showing an abscess measuring $6 \mathrm{~cm}$ (Panel B). A small pulmonary nodule with central cavitation appearing in the left upper lobe on day 13 (Panel C) completely disappeared on repeat CT performed on day 33. CT: computed tomography, MRI: magnetic resonance imaging

neutrophils. Computed tomography (CT) showed a 5-cm abscess in the right corpus cavernosum (Fig. 1A). He was then admitted and started on ceftriaxone (day 0 ).

Blood cultures did not reveal any microorganisms. On day 2 , magnetic resonance imaging showed an abscess measuring $6 \mathrm{~cm}$ (Fig. 1B). Surgical drainage was performed on the same day. Suprapubic percutaneous needle aspiration drained a moderate amount of purulent fluid. A 2-cm skin incision was then made along the perineal raphe to visualize the corpus cavernosum. However, attempts at the perineal approach were not successful. The surgical team decided not to proceed with incision drainage because of the risk of significant cavernosal bleeding. On day 3, a CT-guided drainage tube was added suprapubically. The aspirated material revealed neither microorganisms nor bacteria on Gram staining. Polymerase chain reaction was negative for both Neisseria gonorrhea and Chlamydia trachomatis.

Despite these treatments, the pyrexia and leukemoid reactions persisted, and the patient became increasingly lethargic. The scrotum and penis became diffusely edematous and out of proportion to the surgical invasion, and the incision on the scrotum began to erode. On day 12 , the patient reported poor vision. Both eyes showed prominent episcleritis and findings consistent with those of peripheral ulcerative keratitis. He was barely able to count raised fingers shown to him with his right eye. Two erythematous nodules were noted along a phlebitis on his left forearm near a peripheral catheter insertion point (Fig. 2A). The perineal raphe developed a prominent necrotic ulcer, and another large necrotic ulcer with purpuric margins covered the entire dorsum of his penis, apparently originating from the insertion of the needle aspiration (Fig. 2B). Necrotizing soft tissue infection was suspected, and meropenem was initiated. Debridement of the penis was deferred because the subsequent appearance of necrotic ulcers was consistent with pathergy, a finding suggestive of neutrophilic dermatosis. Systemic colchicine treatment was initiated along with local betamethasone instillation for the ocular lesions.
The patient did not show any significant clinical improvements despite treatment. Pulmonary nodules with central cavitation were noted on CT on day 13 (Fig. 1C). The specimens from the necrotic tissue collected before the initiation of meropenem were sterile. A biopsy of the necrotic ulcer revealed prominent neutrophilic infiltrates without vasculitic findings (Fig. 3). At this point, the patient met the diagnostic criteria for NND proposed by Sanchez et al. (5). Methylprednisolone (1,000 mg) was administered daily from days 14 to 16 , followed by $60 \mathrm{mg}$ of oral prednisolone daily. His ocular lesions and leukemoid reactions showed dramatic responses to treatment. The scrotal and penile lesions also became less purulent and edematous.

The penile and scrotal ulcers became shallow and did not expand; however, hypospadias developed on the ventral part of the external urethral meatus. Cystostomy was performed on day 19. Owing to his partial therapeutic response, the dosage of oral prednisolone was changed to $80 \mathrm{mg}$ methylprednisolone daily, and methylprednisolone pulse therapy was repeated from day 21 to 23 . Thereafter, the dose for methylprednisolone was tapered. Other conditions, including pyrexia, lethargy, and visual disturbance symptoms, all markedly improved. The necrotic ulcers healed with scar formation; however, the penis became progressively gangrenous, and an almost completely dry gangrene developed on day 26 (Fig. 2C). Total penectomy was planned, and the patient was transferred to another hospital for this procedure on day 42.

No underlying conditions of neutrophilic dermatosis were identified during hospitalization. Bone marrow biopsies were repeated three times, none of which were diagnostic. He did not have a history consistent with inflammatory bowel disease, and colonoscopy performed on day 26 showed no abnormalities.

\section{Discussion}

Our case revealed an association between neutrophilic 

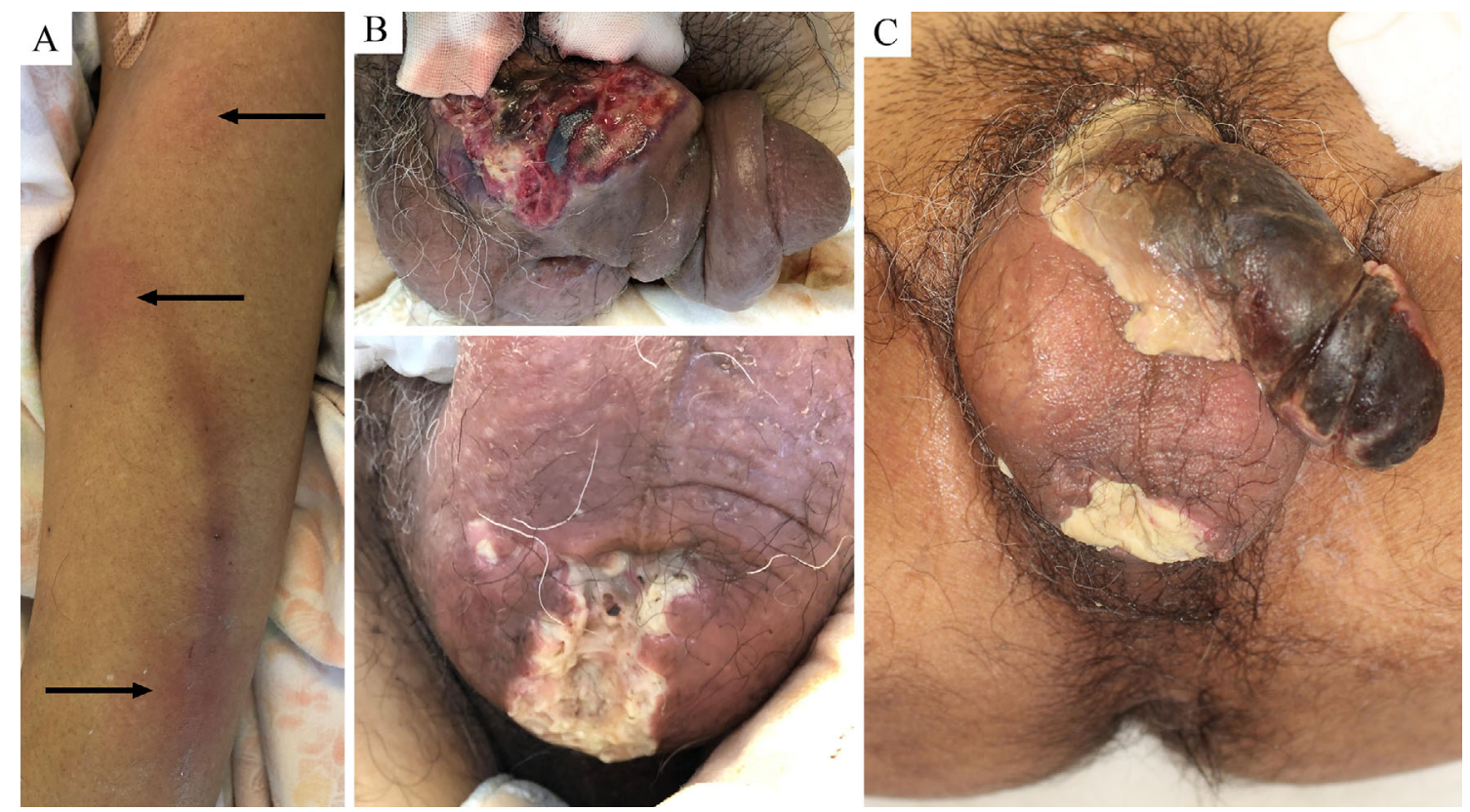

Figure 2. Cutaneous findings of the patient. Cutaneous lesions on the left forearm on day 14 (Panel A). Three painful indurations are visible along an erythematous vein (arrows). Purulent necrotic ulcers can be noted on the dorsal penis and scrotum, where surgical invasion occurred (Panel B). The penis progressively formed dry gangrene, while the surrounding ulcers did not expand further (Panel C).

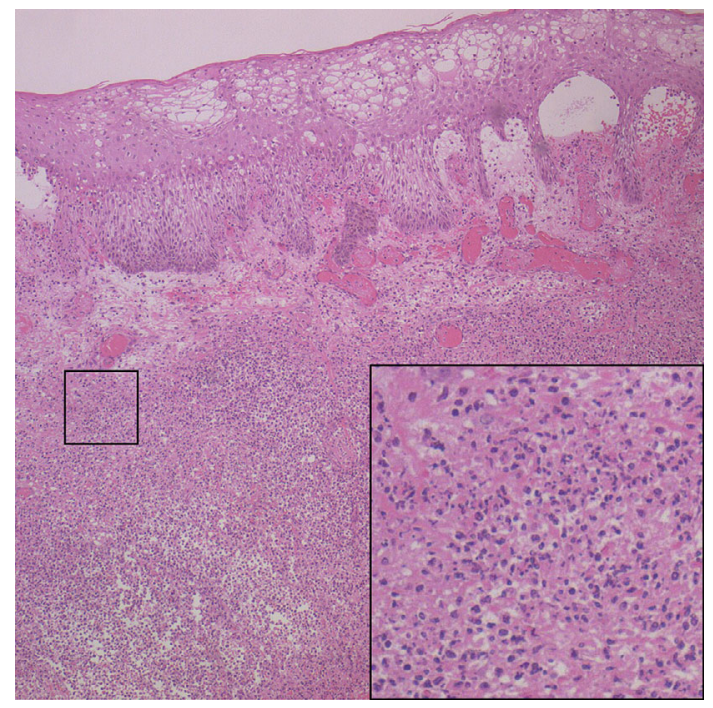

Figure 3. Pathological findings of the penis. Biopsy specimen of the necrotic ulcer showing prominent neutrophilic infiltrate in the dermis and subcutaneous tissue [Hematoxylin and Eosin staining $(50 \times)]$. The square displays an increased magnification (100x).

dermatosis and cavernosal abscess. Although the specimen was obtained from the abscess after the initiation of antibiotics, the abscess itself was considered sterile. A rigorous review of the results of the Gram staining by the physician team was negative. The patient's symptoms did not respond to antibiotic therapy either. These results suggest that the abscess did not have an infectious etiology. It was highly unlikely for NND and a spontaneous bacterial cavernosal ab- scess, two very rare clinical entities, to coincide. We concluded that the cavernosal abscess in our case was sterile and a feature of NND.

An earlier diagnosis may have been possible in this case. The patient did not have any known underlying conditions of aseptic abscess syndrome, such as inflammatory bowel disease or hematologic malignancy. Furthermore, the site of the abscess was not typical of aseptic abscess syndrome, which usually forms abscesses in the liver and spleen. Despite these obstacles to the early diagnosis, the patient showed key findings early on in the course of his treatment. Leukemoid reactions were observed from the day of his initial presentation. His penis and scrotum exhibited an exaggerated inflammatory response to surgical intervention. He also exhibited nonspecific but noticeable mild scleritis before he complained of significant visual disturbances. These findings may have led to the early initiation of systemic corticosteroids. The recognition of this condition remains crucial for the early diagnosis.

We conducted a literature review to characterize the association between neutrophilic dermatosis and cavernosal abscesses. We searched the PubMed/Medline and CINAHL electronic databases using combined keywords. The detailed search strategies and selection process are shown in the supplementary data. A total of 144 articles were identified from the preliminary search. After screening the titles and abstracts, a total of 18 full-text articles were retrieved for this study. Finally, we identified four relevant case reports describing aseptic cavernosal abscess with neutrophilic dermatosis. Relevant clinical information of the included patients' cases is summarized in Table. This review included a total 
Table. Clinical Information of the Five Patients Included in the Literature Review.

\begin{tabular}{|c|c|c|c|c|c|c|c|c|c|c|c|}
\hline Patient & Age & Comorbidities & Abscess culture results & Associated neutrophilic dermatoses & Extracutaneous complications & Immunosupressive agents & $\begin{array}{l}\text { Antibiotics } \\
\text { use }\end{array}$ & $\begin{array}{l}\begin{array}{l}\text { Surgical } \\
\text { debredement or } \\
\text { darinage }\end{array} \\
\text {. }\end{array}$ & Urinary diversion & Penectomy & Reference \\
\hline 1 & 76 & Prostate cancer & Sterile & $\begin{array}{l}\text { Pyoderma gangrenosum with satellite } \\
\text { lesions }\end{array}$ & $\begin{array}{l}\text { Aseptic prostatic and lung } \\
\text { abscesses }\end{array}$ & Prednisolone & Yes & Incision drainage & Illeal conduit & No & (6) \\
\hline 2 & 76 & None & Sterile & Pyoderma gangrenosum & None & Prednisolone, cyclosporine & Yes & Incision drainage & None & No & (7) \\
\hline 3 & 54 & None & $\begin{array}{l}\text { Enterococcus faecalis was } \\
\text { detected as a contaminant. }\end{array}$ & $\begin{array}{l}\text { Pyodemma gangrenosum with satellite } \\
\text { lesions }\end{array}$ & None & Prednisolone & Yes & Debredement & $\begin{array}{l}\text { None (Penis reconstruction } \\
\text { was performed.) }\end{array}$ & $\begin{array}{l}\text { Yes (partial } \\
\text { penectomy) }\end{array}$ & (8) \\
\hline 4 & 67 & None & $\begin{array}{l}\text { Staphylococcus epidermis } \\
\text { was detected as a } \\
\text { contaminant. }\end{array}$ & Pyoderma gangrenosum & None & Prednisolone & Yes & Incision drainage & Cystostomy & No & (9) \\
\hline 5 & 50 & None & Sterile & Necrotizing neutrophilic dermatosis & $\begin{array}{l}\text { Peripheral uleerative keratitis, } \\
\text { lung nodules, skin nodules, } \\
\text { phlebtitis }\end{array}$ & Corticosteroids, colchicine & Yes & Aspiration drainage & Cystostomy & $\begin{array}{l}\text { Yes (Transfered } \\
\text { for penectomy) }\end{array}$ & $\begin{array}{l}\text { Current } \\
\text { ease }\end{array}$ \\
\hline
\end{tabular}

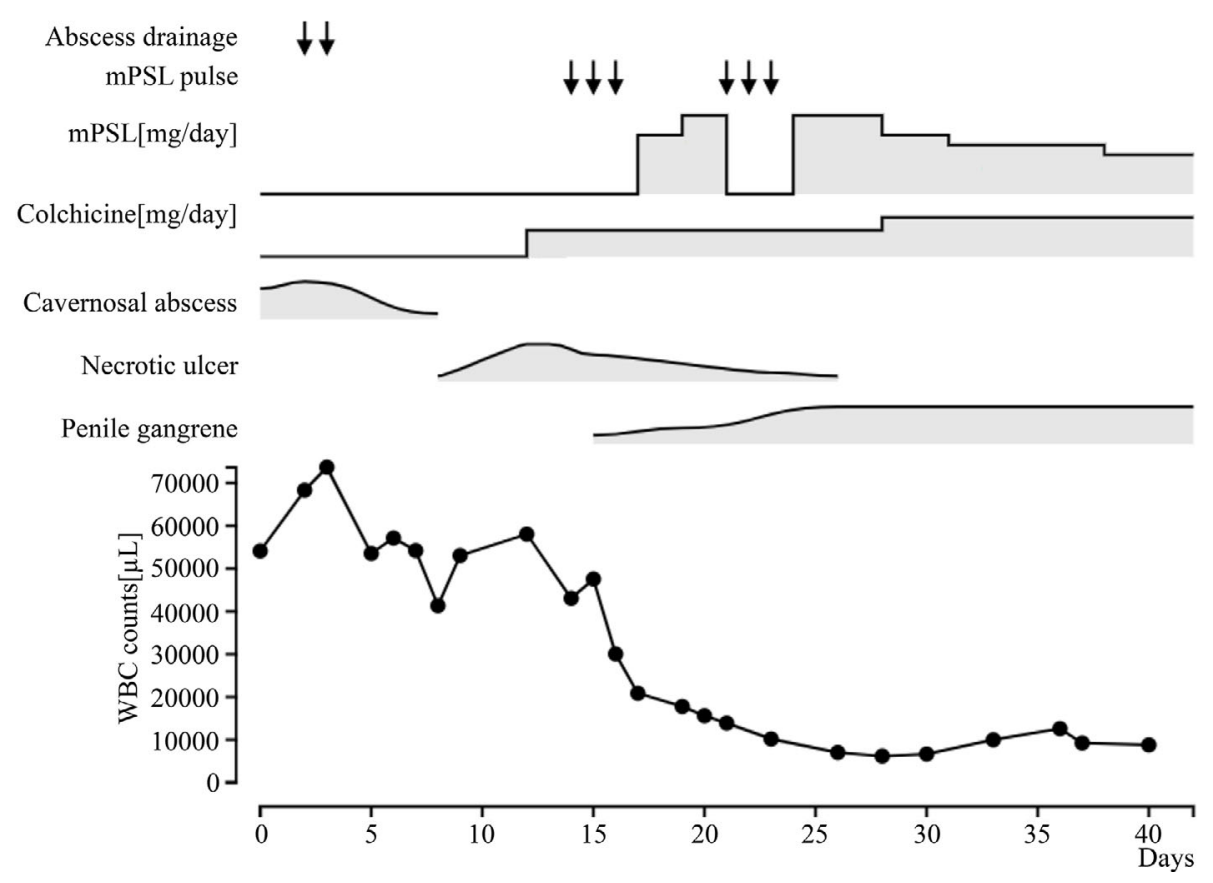

Figure 4. The clinical course of the patient. The observed findings of the cavernosal abscess, necrotic ulcers, and penile gangrene are shown in visual scales with curves reproduced from the patient's medical record. The peaks of the curves indicate the worst clinical observations. The patient was administered oral prednisolone instead of methylprednisolone on days 17 and 18. mPSL: methylprednisolone, WBC: white blood cell

of five patients, including the current case. The mean age at the time of the diagnosis was 65 years old. None of the patients had a diagnosis of neutrophilic dermatoses or other common associated conditions before the detection of a penile abscess. Four patients, excluding our own case, were eventually diagnosed with pyoderma gangrenosum of the penis. All patients underwent surgical intervention and were prescribed antibiotics. The initial abscess cultures did not reveal any significant organisms in any patient. All patients survived, but most underwent penectomy or urinary diversion. Patient 2 did not undergo either procedure; however, his penile lesions healed with a significant deformity. The most frequently administered immunosuppressive agents were corticosteroids. All of the articles reported excellent responses to immunosuppressive therapies, except for with regard to the penile function.

Our review added key clinical information on aseptic cavernosal abscess in our case report. The neutrophilic dermatosis most frequently described with aseptic abscess is pyo- derma gangrenosum (4). These results are comparable to those found in our review. Neutrophilic dermatoses are often accompanied by underlying illnesses, such as hematological malignancies and inflammatory bowel diseases. The patients in our review did not have these common underlying illnesses. Aseptic cavernosal abscesses appeared to be less frequently caused by preexisting underlying conditions. All included articles were Japanese studies. The results yielded no reports from outside Japan, even though we did not apply limitations to our searches. Thus, as a clinical entity, an aseptic cavernosal abscess accompanied by other features of neutrophilic dermatosis may be ethnically predominant.

The pathogenesis of aseptic cavernosal abscesses is unknown. Patient 1 had a concurrent prostatic abscess that developed after radiation therapy for prostate cancer. Our patient, an avid cyclist, presumably had chronic pressure being placed on his perineum. Thus, external stimuli may have been triggers for this condition.

The prognosis of the penile function is poor. Aseptic ab- 
scesses usually respond favorably to corticosteroid therapy without causing significant morbidity (4). In contrast, all of the cases in this report were complicated with significant anatomical destruction of the penis, often necessitating urinary diversion or penile reconstruction. In our case, while most of the features of NND were resolved, the penis eventually formed complete dry gangrene. The poor prognosis of the penis is considered to be related to its anatomical fragility. The corpus cavernosum receives its blood supply from the cavernosal artery which is confined within the tunica albuginea, making it susceptible to ischemia, as seen in priapism $(10,11)$. Abscesses in deep organ structures, such as the liver and spleen, frequently receive unnecessary surgical interventions and antibiotic therapies. Similarly, all cases in our study underwent surgical intervention and were prescribed antibiotics. Bacterial abscesses benefit from these treatments; however, such an approach is considered ineffective for aseptic abscesses, and surgical intervention may precipitate significant local inflammation through pathergy in the presence of neutrophilic dermatoses. Thus, the decision to perform surgical interventions should be made carefully. Considering the poor functional prognosis of the penis, this is of particular importance.

\section{Conclusion}

An aseptic cavernosal abscess is a manifestation of neutrophilic dermatosis. The prognosis of the penile function is poor. This condition is often mistaken for bacterial abscesses, and pathergic reactions to surgical interventions may worsen the condition. Meticulous searches for key features of neutrophilic dermatosis, such as leukemoid reaction, a history of pathergy, satellite lesions, or common underlying comorbidities, are essential for the early diagnosis, which can potentially improve the functional prognosis of the penis. Clinicians must therefore be aware of this condition to distinguish it from bacterial infections.

\section{The authors state that they have no Conflict of Interest (COI).}

\section{Acknowledgement}

We thank the ward staff and members of our infectious disease control division for their devotion during the coronavirus disease 2019 pandemic. We are also grateful to Yoshimi Kanda for providing technical advice.

\section{Supplementary data}

We searched articles published up to March 9, 2021, and indexed in PubMed/Medline and CINAHL electronic databases with multiple combined keywords. The following search strategy was used for PubMed/Medline: ((Penile diseases[MeSH Terms]) OR (Penis[MeSH Terms]) OR (Penis[Text Word]) OR (Penile [Text Word]) OR (Corporal[Text Word]) OR (Cavernos*[Text Word]) OR (Periurethral[Text Word]) OR (Peri-urethral[Text Word])) AND ((Abscess[MeSH Terms]) OR (Abscess*[Text Word])) AND ((Autoimmune diseases[MeSH Terms]) OR (Skin and Connective tissue diseases[MeSH Terms]) OR (Immunosuppressive Agents[MeSH Terms]) OR (Autoimmun*[Text Word]) OR (Pyoderma gangrenosum[Text Word]) OR (Pathergy[Text Word]) OR (Sweet[Text Word]) OR (Sweet's[Text Word]) OR (Behcet[Text Word]) OR (Behcet's[Text Word]) OR (Neutrophilic [Text Word]) OR (Steroid*[Text Word]) OR (Corticosteroid* [Text Word]) OR (Glucocorticoid*[Text Word]) OR (Cyclosporine[Text Word]) OR (Predniso*[Text Word]) OR (Immunosuppress*[Text Word]))

The titles and abstracts of all identified articles were screened, and the full texts of the identified articles that passed the title and abstract screening were reviewed for inclusion in the review. Case series and case reports of cavernosal abscesses associated with neutrophilic dermatoses were included in the study. Cases in which significant microorganisms were detected from either the abscesses or the peripheral blood were excluded. Clinical data were then extracted from the included articles. Each step was independently performed by two reviewers (Y. A. and Y. Y.). EndNote X9 and Microsoft Excel spreadsheets were used for the entire selection process and data collection, respectively.

\section{References}

1. Dugdale CM, Tompkins AJ, Reece RM, Gardner AF. Cavernosal abscess due to Streptococcus anginosus: A case report and comprehensive review of the literature. Curr Urol 7: 51-56, 2013.

2. Paladino JR, Nascimento FJ, Gromatsky C, Pompeo AC. Corpus cavernosum abscess after winter procedure performance. BMJ Case Rep 2014: bcr2013202089, 2014.

3. Gore TC, Schepcoff A, Sorresso D. Corpus cavernosum abscess secondary to traumatic perforation of urethral diverticulum. Cureus 12: e7032, 2020.

4. André MFJ, Piette JC, Kémény JL, et al. Aseptic abscesses: a study of 30 patients with or without inflammatory bowel disease and review of the literature. Medicine (Baltimore) 86: 145-161, 2007.

5. Sanchez IM, Lowenstein S, Johnson KA, et al. Clinical features of neutrophilic dermatosis variants resembling necrotizing fasciitis. JAMA Dermatol 155: 79-84, 2019.

6. Kanno T, Ito M, Tsuji H, Kawase N, Taki Y. A case of pyoderma gangrenosum involving the prostate gland after radiation therapy for prostate cancer. Hinyokika Kiyo 48: 565-568, 2002 (in Japanese).

7. Iida K, Mizuno K, Kawai N, et al. A case of abscess of corpus cavernosum as an early symptom of penile pyodermal gangrenosum: We propose the possibility of a new pathogenic finding. Hinyokika Kiyo 61: 115-119, 2015 (in Japanese).

8. Taguchi M, Inoue $T$, Nishida $T$, et al. A case of pyoderma gangrenosum of the penis difficult to distinguish from Fournier gangrene. Hinyokika Kiyo 61: 459-463, 2015 (in Japanese).

9. Takahashi S, Numakura K, Kubo K, et al. A case of penile pyoderma gangrenosum treated with steroid administration without penectomy. Hinyokika Kiyo 65: 219-222, 2019 (in Japanese).

10. Anele UA, Le BV, Resar LM, Burnett AL. How I treat priapism. Blood 125: 3551-3558, 2015.

11. Melman A, Serels S. Priapism. Int J Impot Res 12 (Suppl 4): S133-S139, 2000.

The Internal Medicine is an Open Access journal distributed under the Creative Commons Attribution-NonCommercial-NoDerivatives 4.0 International License. To view the details of this license, please visit (https://creativecommons.org/licenses/ by-nc-nd/4.0/). 
(C) The Japanese Society of Internal Medicine Intern Med Advance Publication 